\title{
Color and Metallicity Distributions of M81 Globular Clusters
}

\author{
Jun $\mathrm{Ma}^{1}, \mathrm{Xu}$ Zhou${ }^{1}$, Jiansheng Chen ${ }^{1}$, Zhenyu Wu${ }^{1}$, Yanbin Yang ${ }^{1}$, Zhaoji Jiang ${ }^{1}$, \\ Jianghua $\mathrm{Wu}^{1}$ \\ majun@vega.bac.pku.edu.cn
}

\begin{abstract}
In this paper we present catalogs of photometric and spectroscopic data for M81 globular clusters (GCs). The catalogs include $B-$ and $V$ - photometric and reddening data of 95 GCs, and spectroscopic metallicities of $40 \mathrm{GCs}$ in M81. Using these data, we make some statistical correlations. The results show that the distributions of intrinsic $B$ and $V$ colors and metallicities are bimodal, with metallicity peaks at $[\mathrm{Fe} / \mathrm{H}] \approx-1.45$ and -0.53 , respectively as has been demonstrated for our Milky Way and M31. The relation between spectroscopic metallicity and intrinsic $B$ and $V$ color also exists as it does for the Milky Way and M31.
\end{abstract}

Subject headings: galaxies: individual (M81) - galaxies: star clusters - globular clusters: general

\section{Introduction}

Great progress has been made in the past decade in our understanding of globular cluster systems (GCSs) of galaxies, especially the discovery that many galaxies possess two or more distinct subpopulations of globular clusters (GCs) (e.g., West et al. 2004, and references therein). GCs are fossils of the earliest stages of galaxy formation, and are groups of stellar populations with a single age and metallicity. Consequently, their integrated properties, such as abundance and kinematics, can provide us with valuable information about the nature and duration of the formation of their parent galaxies (Barmby et al. 2000). The metallicity distribution of GCs is of particular importance in deepening our knowledge of the dynamical and chemical evolution of the parent galaxies. For example, the GCs of many elliptical galaxies show multi-modal metallicity distributions, suggesting that multiple star formation episodes occurred in these elliptical galaxies in the past (Zepf \& Ashman 1993; Barmby et al. 2000).

Côté (1999) presents a metallicity distribution of 133 Galactic GCs that apparently shows two peaks (i.e., two distinct metal-poor and metal-rich GC populations). The double-Gaussian can best fit these two subpopulations, the mean metallicity values are -1.59 and -0.55 dex, respectively. Using the data for 247 GCs in M31, Barmby et al.

\footnotetext{
${ }^{1}$ National Astronomical Observatories, Chinese Academy of Sciences, Beijing, 100012, P. R. China; majun@vega.bac.pku.edu.cn
}

(2000) studied the metallicity distribution, which is asymmetric, implying the possibility of bimodality. Then the applied KMM algorithm showed that the metallicity distribution is really bimodal. Perrett et al. (2002) confirmed the conclusions of Barmby et al. (2000).

M81 is the nearest large spiral outside the Local Group, and its GCs are an important target of study. However, to date, there are only a few papers that studied the GCs in M81. The main reason is that at the distance of M81, the diameters of GCs are typically comparable to the seeing disk, and it is difficult to recognize GCs on the basis of image structure from ground-based observations, except at sites with exceptionally good seeing. To maximize the success rate of the GC candidate list for the ongoing spectroscopic observations, Perelmuter \& Racine (1995) used an extensive database that included photometric, astrometric, and morphological information on 3774 objects covering over a $>50$ arcmin diameter field centered on M81 to reveal 70 GC candidates within $11 \mathrm{kpc}$ galactocentric radius. Perelmuter, Brodie, \& Huchra (1995) then confirmed 25 bona fide M81 GCs from the spectroscopy of 82 bright GC candidates in the M81 field. Schroder et al. (2002) presented moderate-resolution spectroscopy for 16 GC candidates from the list in Perelmuter \& Racine (1995), and confirmed these 16 candidates as bona fide GCs. They also obtained metallicities for 15 of the 16 GCs. With the superior resolution of the Hubble Space Telescope $(H S T)$, M81 is close enough for its clusters to be easily resolved on the basis of image structure 
(Chandar, Ford, \& Tsvetanov 2001). Thus, using the $B, V$, and $I$ bands of $H S T$ Wide Field Planetary Camera 2 (WFPC2), Chandar, Ford, \& Tsvetanov (2001) imaged eight fields covering a total area of $\sim 40 \operatorname{arcmin}^{2}$. They reported 114 compact star clusters in M81, 59 of which are GCs.

The outline of the paper is as follows. Details of collecting GCs are given in $\S 2$. In $\S 3$ we provide some statistical relationships. The summary and discussion are presented in $\S 4$.

\section{A Catalog of Photometric Data of Globular Clusters}

\subsection{Sample of Globular Clusters}

The sample of GCs of M81 in this paper is from Perelmuter, Brodie, \& Huchra (1995); Schroder et al. (2002); Chandar, Ford, \& Tsvetanov (2001). Perelmuter, Brodie, \& Huchra (1995) obtained spectra for 82 bright GC candidates in the M81 field and confirmed 25 bona fide GCs. Schroder et al. (2002) observed moderate-resolution spectroscopy of 16 GC candidates selected from the candidate list of Perelmuter \& Racine (1995) and confirmed all as bona fide GCs. Chandar, Ford, \& Tsvetanov (2001) discovered 114 compact star clusters (as mentioned above), 59 of them confirmed GCs. Clusters Id50552, Is50696, and Id50826 of Perelmuter, Brodie, \& Huchra (1995) are numbers 87, 40 and 8 of Chandar, Ford, \& Tsvetanov (2001), and objects 13 and 15 of Schroder et al. (2002) are 1 and 7 of Chandar, Ford, \& Tsvetanov (2001), respectively. Altogether, there are $95 \mathrm{GCs}$, which are listed in Table 1 [col. (1) is the name of the GC, and cols. (2) and $(3)$ are the $V$-band magtitude and $(B-V)_{0}$ color, respectively].

\subsection{Reddening}

In order to obtain intrinsic colors for the GCs, in addition to the absolute magnitudes, the photometric data should be corrected for reddening from the foreground extinction contribution of the Milky Way and for the internal reddening due to varying optical paths through the disk of M81. The total reddening in M81 (foreground plus M81 contribution) has been measured by a number of authors (e.g., Freedman, Wilson, \& Madore 1994; Kong et al. 2000). We only mention here that Kong et al. (2000) obtained the reddening maps of M81 based on the images in 13 intermediate-band filters from 3800 to $10000 \AA$. To determine the metallicity, age, and reddening distributions for M81, Kong et al. (2000) found the best match between the observed colors and the predictions from the single stellar population models of Bruzual \&
Charlot (1996). A map of the interstellar reddening in a substantial portion of M81 were obtained. We used the reddening data of Kong et al. (2000). For a few clusters that fall near the edges of the images, Kong et al. (2000) did not present the reddening data, we adopt the mean reddening value of 0.13 as Chandar, Ford, \& Tsvetanov (2001) did. Since Chandar, Ford, \& Tsvetanov (2001) also used the reddening data of Kong et al. to deredden their star clusters, we only deredden the GCs of Perelmuter, Brodie, \& Huchra (1995) and Schroder et al. (2002). These local reddening values are listed in column (4) of Table 1 . For completeness, we also list the reddening data for the GCs of Chandar, Ford, \& Tsvetanov (2001).

\section{Properties of Globular Clusters}

\subsection{Luminosity Function and Color Mag- nitude Diagram}

We show in Figure 1 the luminosity functions for all the sample GCs in M81. The magnitudes are corrected for extinction based on the local $E_{(B-V)}$ value given in column (3) of Table 1 and the optical Galactic extinction law with $R_{v}=3.1$ and a distance modulus for M81 of 27.8 (Freedman, Wilson, \& Madore 1994; Chandar, Ford, \& Tsvetanov 2001). We can see that the M81 GC luminosity function is not unimodal (without an apparent peak). However, with a fainter completeness limit, the turnover may appear.

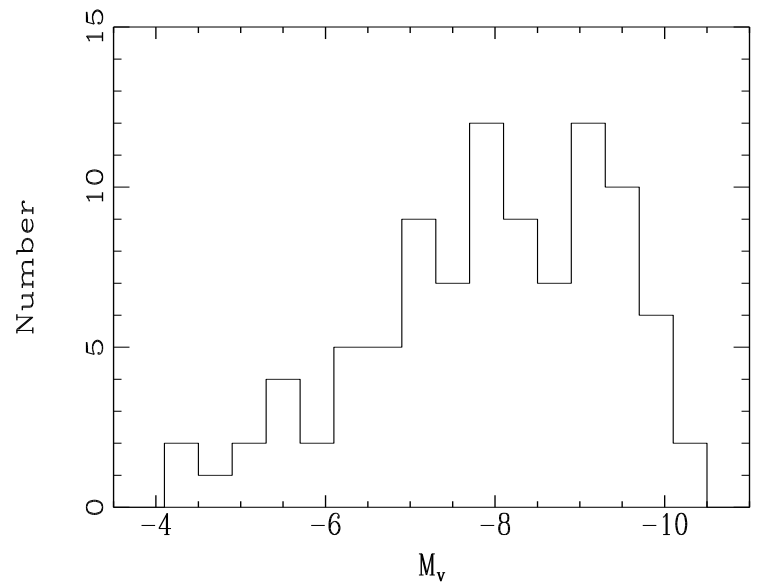

Fig. 1. - Luminosity function of the sample globular clusters.

Figure 2 plots a $(B-V)_{0}$ versus $M_{V}$ colormagnitude diagram for the M81 GCs. Colors have been dereddened by the local $E_{(B-V)}$ value given in column (3) of Table 1. From Figure 2, we note that there are some GCs that have $(B-V)_{0}$ greater than 1.0. By comparing with the $(B-V)_{0}$ 
of Galactic GCs (Harris \& Racine 1979) and M31 GCs (Barmby et al. 2000), these M81 GCs have great $(B-V)_{0}$. These red colors may result from enhanced foreground reddening comparing with the applied $E_{(B-V)}$. In particular, there is a GC (96 of Chandar, Ford, \& Tsvetanov 2001) having the $(B-V)_{0}=1.778$, which is too high.

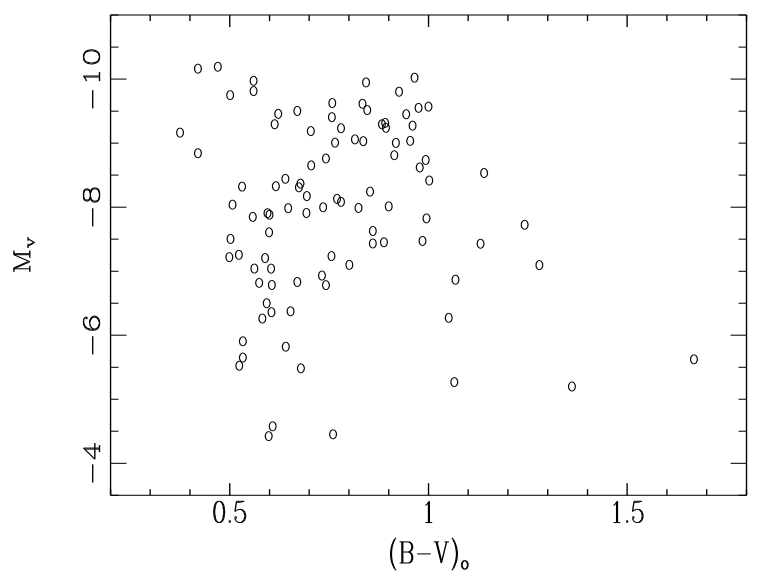

Fig. 2.- Color-magnitude diagrams of the sample globular clusters.

Figure 3 shows the $(B-V)_{0}$ histogram for our sample globular clusters, and two color peaks appear clearly. Using deep F555W and F814W images from WFPC2 on board the HST, Kundu \& Whitmore (2001) presented the results of the GC systems of 28 elliptical galaxies, in which the $V-I$ color distributions of at least $50 \%$ of the sample galaxies appear to be bimodal. In addition, from $H S T$ imaging in $555 \mathrm{~W}$ and $\mathrm{F} 814 \mathrm{~W}$ filters, Larsen et al. (2001) studied the GCs in 17 relatively nearly early-type galaxies and found that a sum of two Gaussians provides a better fit to the observed color distribution than a single Gaussian. To make quantitative statements about the bimodality of $(B-V)_{0}$ in this paper, a KMM test (Ashman, Bird, \& Zepf 1994) is applied to the data. This test uses a maximum likelihood method to estimate the probability that the data distribution is better modeled as a sum of two Gaussians than as a single Gaussian. Here we use a homoscedastic test (i.e., the two Gaussians are assumed to have the same dispersion). The $(B-V)_{0}$ of the two peaks, the $P$-value, and the numbers of GCs assigned to each peak by the KMM test are $(B-V)_{0} \approx 0.98$ and $0.66,0.071$, and 67 and 27 . The $P$-value is in fact the probability that the data are drawn from a single Gaussian distribution. Since the GC 96 of Chandar, Ford, \& Tsvetanov (2001) has very high $(B-V)_{0}[=1.778]$, we do not include it when conducting the KMM test. We note that the second Gaussian peak looks like a terrible fit, which may be because the data sample is not large enough. Barmby et al. (2000) analyzed the distribution of 10 intrinsic colors for the 221 M31 GCs and tested the color distributions of these GCs for bimodality using the KMM algorithm (Ashman, Bird, \& Zepf 1994); the peaks of the $(B-V)_{0}$ are 0.83 and 0.68 . The peaks of the other intrinsic color distributions are presented in Table 8 of Barmby et al. (2000). For the color distributions of the Galactic GCs, Barmby et al. (2000) only plotted the distribution of $(V-I)_{0}$, and did not apply the KMM test. From Figure 14 of Barmby et al. (2000), two clear peaks of distribution of $(V-I)_{0}$ can be seen.

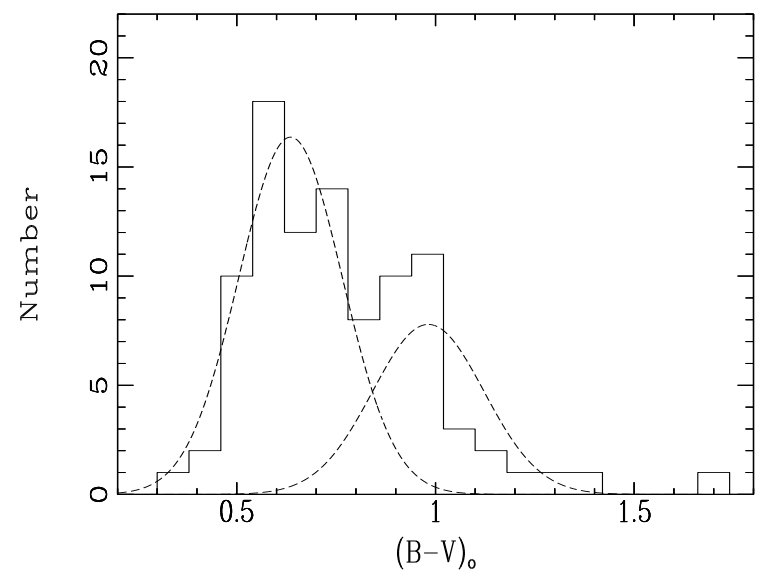

Fig. 3.- Color histogram distribution of the sample globular clusters.

\subsection{Metallicity Distribution of the Sam- ple Globular Clusters Using Only with Spectroscopic Data}

Perelmuter, Brodie, \& Huchra (1995) obtained the spectra for 25 M81 GCs with the Hydra multifiber positioner and bench spectrograph on the KPNO $4 \mathrm{~m}$ telescope. Schroder et al. (2002) then observed 16 M81 GCs with the Low Resolution Imaging Spectrograph on the Keck I telescope and presented the moderate-rosolution spectroscopy for these GCs, in addition to the metallicities for 15 of the 16 GCs. There is one GC, number 12 of Schroder et al. (2002), for which the metallicity was not obtained, since a transient phenomenon was occurring in this GC at the time of the observation. Thus, there are a total of 40 GCs for which we have the metallicities, which are listed in Table 2. However, some of Perelmuter, Brodie, \& Huchra (1995) sample GC metallicities have large uncertainties, which can create uncertainties in the metallicity distributions. Therefore, 
Figure 4 only shows the metallicity diagram of 26 M81 GCs with metallicity uncertainties smaller than 1.0 dex. Figure 4 does not show two peaks, and perhaps because the number of sample GCs are too small. In order to enlarge the number of GCs, we use metallicities estimated from colors by the color-metallicity relation.

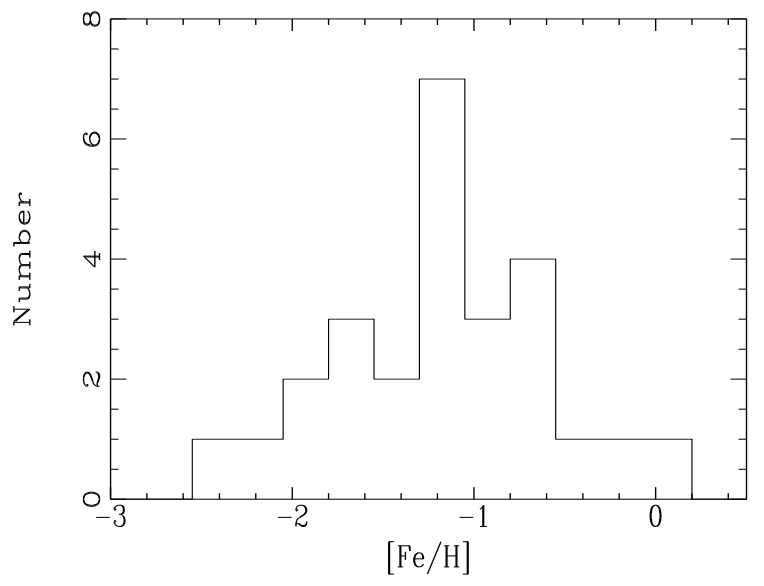

Fig. 4.- Metallicity diagram for the globular clusters only with spectroscopic data.

\subsection{Color-Metallicity Correlation}

Originally, Brodie \& Huchra (1990) derived the correlation between the IR colors and metallicity of 23 low-reddening Galactic GCs. Kissler-Patig et al. (1998) studied the GCs of NGC 1399 using moderate-resolution, high signal-to-noise ratio spectroscopy and found that $V-I$ and metallicity are well correlated. To determine the reddening and use the color to predict metallicity, Barmby et al. (2000) performed linear regressions of the color-metallicity for M31 and Galactic GCs.

We present below the relation between intrinsic color in the $B$ and $V$ bands and metallicity for 26 M81 GCs for which we have spectroscopic metallicities with metallicity uncertainties smaller than 1.0 dex. We do an ordinary least-squares fit:

$$
[\mathrm{Fe} / \mathrm{H}]=a(B-V)_{0}+b
$$

Figure 5 shows this fit. The fit results are $a=$ $2.41 \pm 0.46$ and $b=-3.08 \pm 0.37$, and the linear correlation coefficient $r=0.73$.

Couture, Harris, \& Allwright (1990) established the intrinsic $B-V$ color-metallicity relation for 65 Galactic GCs, using the data from Reed, Hesser, \& Shawl (1988) and Zinn (1985), for which the foreground reddening is low $\left(E_{B-V} \leq 0.4\right)$ and reasonably well known:

$$
(B-V)_{0}=0.200[\mathrm{Fe} / \mathrm{H}]+0.971
$$

Barmby et al. (2000) also presented the intrinsic $B-V$ color-metallicity relation for 88 Galactic GCs with $E_{B-V}<0.5$ (the database of Galactic GC parameters were from the 1999 June version of the Harris (1996)):

$$
(B-V)_{0}=0.159 \pm 0.011[\mathrm{Fe} / \mathrm{H}]+0.92 \pm 0.02
$$

In order to make comparisons with the intrinsic $B-V$ color-metallicity relation for the Galactic GCs, we establish this relation using the data from the 26 M81 GCs used in Figure 5:

$$
(B-V)_{0}=0.222 \pm 0.042[\mathrm{Fe} / \mathrm{H}]+1.05 \pm 0.05
$$

We can see that the intrinsic $B-V$ colormetallicity relation between the Galactic GCs and the M81 GCs is consistent by comparing equations (2), (3) and (4).

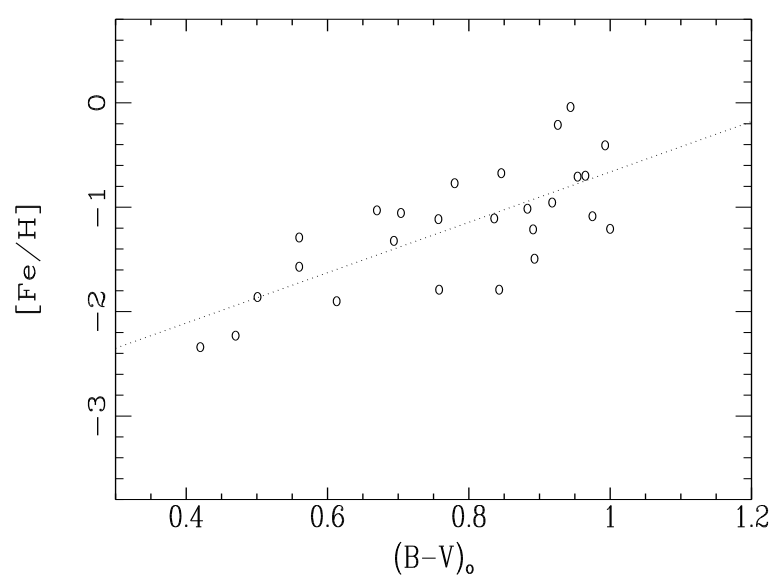

Fig. 5.- Color in $B V$ bands vs. spectroscopic metallicities for M81 globular clusters.

\subsection{Metallicity Distribution of All the Sample Globular Clusters}

We use the above color-metallicity correlation to derive metallicities for the sample GCs that do not have spectroscopic metallicities or that have metallicity uncertainties larger than 1 dex. We plot metallicity distribution in Figure 6, including the metallicities derived using the above color-metallicity correlation. To make quantitative statements about bimodality, we also apply a KMM test (Ashman, Bird, \& Zepf 1994) to the data. The metallicities of the two peaks, the $P$ value, and the numbers of clusters assigned to each 
peak by the KMM test are $[\mathrm{Fe} / \mathrm{H}] \approx-1.45$ and $-0.54,0.024$, and 74 and 20, respectively. Since the GC 96 of Chandar, Ford, \& Tsvetanov (2001) has very high $(B-V)_{0}\left((B-V)_{0}=1.778\right)$, and the metallicity obtained using the color-metallicity correlation is too rich (0.95 dex), we do not include it when performing ing the KMM test.

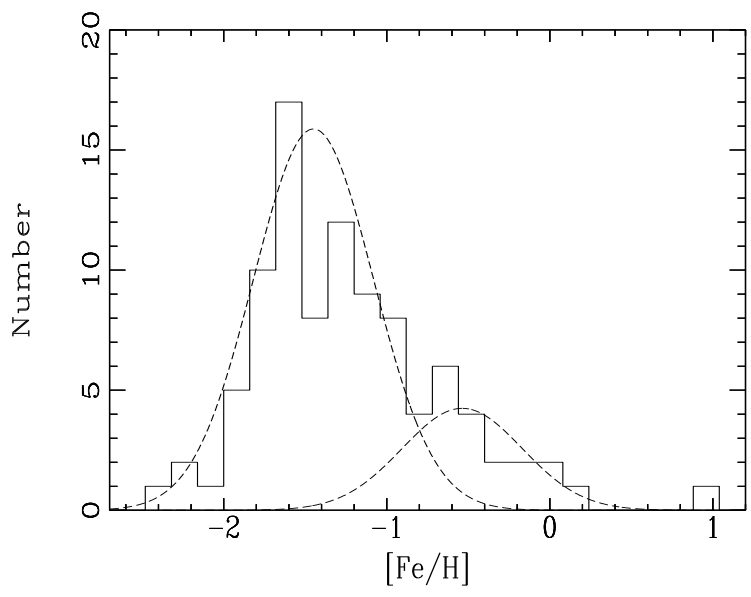

Fig. 6.- Metallicity diagram for all the globular clusters.

\section{SUMMARY AND DISCUSSION}

In this paper, we present catalogs of photometric and spectroscopic data and statistical correlations for M81 GCs. Using the KMM test, we find that the distribution of intrinsic $B$ and $V$ colors and metallicities are bimodal. The correlation between spectroscopic metallicity and intrinsic $B$ and $V$ color also exists as it does for the Milky Way and M31.

We would like to thank the anonymous referee for his/her insightful comments and suggestions that improved this paper very much. This work has been supported by the Chinese National Key Basic Research Science Foundation (NKBRSF TG199075402) and in part by the Chinese National Science Foundation, No. 10473012.

\section{REFERENCES}

Ashman, K. A., Bird, C. M., \& Zepf, S. E. 1994, AJ, 108, 2348

Barmby, P., Huchra, J., Brodie, J., Forbes, D., Schroder, L., \& Grillmair, C. 2000, AJ, 119, 727

Brodie, J. P., \& Huchra, J. P. 1990, ApJ, 362, 503

Bruzual, G., \& Charlot, S. 1996, unpublished
Chandar, R., Ford, H. C., \& Tsvetanov, Z. 2001, AJ, 122, 1330

Côté, P. 1999, AJ, 118, 406

Couture, J., Harris, W. E., \& Allwright, J. W. B. 1990, ApJS, 73, 671

Freedman, W. L., Wilson, C. D., \& Madore, B. F. 1994, ApJ, 427, 628

Harris, W. E., \& Racine, R. 1979, ARA\&A, 17, 241

Harris, W. H. 1996, AJ, 112, 1487

Kong, X., et al., 2000, AJ, 119, 2745

Kissler-Patig, M., Brodie, J. P., Schroder, L. L., Forbes, D. A., Grillmair, C. J., \& Huchra, J. P. 1998, AJ, 115, 105

Kundu, A., \& Whitmore, B. C. 2001, AJ, 121, 2974

Larsen, S. S., et al., 2001, AJ, 121, 2974

Perelmuter, J. M., Brodie, J. P., \& Huchra, J. 1995, AJ, 110, 620

Perrett, K. M.. et al., 2002, AJ, 123, 2490

Perelmuter, J. M., \& Racine, R. 1995, AJ, 109, 1055

Reed, B. C., Hesser, J. E., \& Shawl, S. J. 1988, PASP, 100,545

Schroder, L. L., Brodie, J. P., Kissler-Patig, M., Huchra, J. P., Phillips, A. C. 2002, AJ, 123, 2473

West, M. J., Côté, P., Marzke, R. O., \& Jordan, A. 2004, Nature, 427,31

Zepf, S. E., \& Ashman, K. A. 1993, MNRAS, 264, 611

Zinn, R. 1985, ApJ, 293, 424
This 2-column preprint was prepared with the AAS LATEX macros v 5.2 
TABLE 1

Globular Cluster SAMPle AND PROPERTIES

\begin{tabular}{|c|c|c|c|}
\hline $\mathrm{ID}^{\mathrm{a}}$ & $\mathrm{V}$ (mag) & $B-V(\mathrm{mag})$ & $E_{B-V}(\mathrm{mag})$ \\
\hline Id30244 & 19.76 & 0.77 & 0.130 \\
\hline Is40083 & 18.39 & 0.69 & 0.130 \\
\hline Is40165 & 18.23 & 0.69 & 0.130 \\
\hline Is40181 & 18.93 & 1.09 & 0.130 \\
\hline Is50037 & 18.04 & 0.55 & 0.130 \\
\hline Is 50225 & 18.43 & 0.97 & 0.026 \\
\hline Is50233 & 19.18 & 0.89 & 0.125 \\
\hline Is 50286 & 20.16 & 0.89 & 0.216 \\
\hline Id50357 & 19.67 & 1.27 & 0.130 \\
\hline Is50394 & 19.24 & 0.57 & 0.195 \\
\hline Id50401 & 19.93 & 1.22 & 0.242 \\
\hline Id50415 & 19.24 & 0.85 & 0.237 \\
\hline Id50785 & 19.08 & 0.86 & 0.238 \\
\hline Is50861 & 19.69 & 0.88 & 0.175 \\
\hline Is 50886 & 18.06 & 0.91 & 0.067 \\
\hline Id50960 & 18.49 & 0.86 & 0.102 \\
\hline Is51027 & 19.36 & 0.55 & 0.130 \\
\hline Is60045 & 18.70 & 0.80 & 0.130 \\
\hline Id70319 & 20.77 & 0.99 & 0.130 \\
\hline Id70349 & 20.12 & 0.91 & 0.130 \\
\hline Is80172 & 18.97 & 0.91 & 0.130 \\
\hline Is90103 & 18.01 & 0.60 & 0.130 \\
\hline SBKHP1 & 18.54 & 1.10 & 0.100 \\
\hline SBKHP2 & 18.97 & 1.02 & 0.066 \\
\hline SBKHP3 & 18.35 & 1.04 & 0.114 \\
\hline SBKHP4 & 19.24 & 1.05 & 0.057 \\
\hline SBKHP5 & 18.45 & 1.04 & 0.065 \\
\hline SBKHP6 & 18.80 & 0.97 & 0.077 \\
\hline SBKHP7 & 19.05 & 1.00 & 0.082 \\
\hline SBKHP8 & 18.01 & 1.04 & 0.075 \\
\hline SBKHP9 & 18.76 & 0.98 & 0.089 \\
\hline SBKHP10 & 20.08 & 0.84 & 0.146 \\
\hline SBKHP11 & 18.59 & 0.82 & 0.063 \\
\hline SBKHP12 & 18.70 & 1.00 & 0.166 \\
\hline SBKHP14 & 19.03 & 0.92 & 0.084 \\
\hline SBKHP16 & 18.73 & 0.99 & 0.144 \\
\hline CFT1 & $19.170 \pm 0.006$ & $0.884 \pm 0.020$ & 0.180 \\
\hline CFT5 & $19.826 \pm 0.008$ & $1.184 \pm 0.029$ & 0.270 \\
\hline CFT6 & $20.263 \pm 0.010$ & $0.813 \pm 0.035$ & 0.120 \\
\hline CFT7 & $18.785 \pm 0.004$ & $0.973 \pm 0.015$ & 0.090 \\
\hline CFT8 & $20.191 \pm 0.008$ & $1.030 \pm 0.025$ & 0.130 \\
\hline CFT15 & $22.462 \pm 0.065$ & $0.584 \pm 0.130$ & 0.060 \\
\hline CFT16 & $22.258 \pm 0.056$ & $0.731 \pm 0.149$ & 0.090 \\
\hline CFT20 & $21.340 \pm 0.026$ & $0.790 \pm 0.085$ & 0.120 \\
\hline CFT21 & $21.860 \pm 0.034$ & $0.793 \pm 0.109$ & 0.140 \\
\hline CFT22 & $20.668 \pm 0.020$ & $1.095 \pm 0.105$ & 0.110 \\
\hline
\end{tabular}


TABLE 1-Continued

\begin{tabular}{|c|c|c|c|}
\hline $\mathrm{ID}^{\mathrm{a}}$ & $\mathrm{V}(\mathrm{mag})$ & $B-V(\mathrm{mag})$ & $E_{B-V}(\mathrm{mag})$ \\
\hline CFT28 & $23.688 \pm 0.131$ & $0.870 \pm 0.163$ & 0.110 \\
\hline CFT30 & $20.649 \pm 0.015$ & $1.094 \pm 0.053$ & 0.270 \\
\hline CFT31 & $22.086 \pm 0.053$ & $1.231 \pm 0.208$ & 0.180 \\
\hline CFT32 & $20.478 \pm 0.018$ & $1.372 \pm 0.085$ & 0.130 \\
\hline CFT34 & $21.029 \pm 0.023$ & $0.906 \pm 0.072$ & 0.150 \\
\hline CFT37 & $19.882 \pm 0.009$ & $0.661 \pm 0.013$ & 0.130 \\
\hline CFT38 & $19.835 \pm 0.006$ & $0.808 \pm 0.012$ & 0.130 \\
\hline CFT39 & $19.319 \pm 0.005$ & $0.832 \pm 0.009$ & 0.090 \\
\hline CFT40 & $18.298 \pm 0.002$ & $0.581 \pm 0.004$ & 0.080 \\
\hline CFT41 & $19.721 \pm 0.006$ & $0.696 \pm 0.009$ & 0.080 \\
\hline CFT42 & $20.033 \pm 0.011$ & $0.717 \pm 0.014$ & 0.070 \\
\hline CFT43 & $21.657 \pm 0.048$ & $0.675 \pm 0.057$ & 0.070 \\
\hline CFT44 & $21.325 \pm 0.027$ & $0.706 \pm 0.051$ & 0.100 \\
\hline CFT45 & $20.534 \pm 0.015$ & $0.709 \pm 0.018$ & 0.110 \\
\hline CFT46 & $20.905 \pm 0.016$ & $0.689 \pm 0.021$ & 0.100 \\
\hline CFT49 & $21.911 \pm 0.054$ & $0.702 \pm 0.079$ & 0.120 \\
\hline CFT51 & $20.681 \pm 0.014$ & $1.231 \pm 0.035$ & 0.100 \\
\hline CFT53 & $20.947 \pm 0.016$ & $0.653 \pm 0.024$ & 0.130 \\
\hline CFT56 & $20.259 \pm 0.010$ & $0.710 \pm 0.014$ & 0.110 \\
\hline CFT58 & $23.065 \pm 0.090$ & $1.511 \pm 0.142$ & 0.150 \\
\hline CFT62 & $19.051 \pm 0.004$ & $0.915 \pm 0.007$ & 0.100 \\
\hline CFT63 & $21.230 \pm 0.026$ & $0.654 \pm 0.260$ & 0.080 \\
\hline CFT65 & $20.668 \pm 0.027$ & $0.622 \pm 0.033$ & 0.120 \\
\hline CFT66 & $20.133 \pm 0.017$ & $0.627 \pm 0.032$ & 0.120 \\
\hline CFT67 & $21.209 \pm 0.035$ & $0.842 \pm 0.038$ & 0.110 \\
\hline CFT68 & $21.070 \pm 0.020$ & $0.704 \pm 0.025$ & 0.100 \\
\hline CFT74 & $21.038 \pm 0.019$ & $0.652 \pm 0.025$ & 0.090 \\
\hline CFT75 & $20.514 \pm 0.011$ & $0.795 \pm 0.019$ & 0.200 \\
\hline CFT76 & $20.890 \pm 0.014$ & $0.599 \pm 0.019$ & 0.100 \\
\hline CFT80 & $22.521 \pm 0.036$ & $0.653 \pm 0.063$ & 0.120 \\
\hline CFT83 & $23.686 \pm 0.061$ & $0.698 \pm 0.117$ & 0.100 \\
\hline CFT85 & $23.532 \pm 0.046$ & $0.708 \pm 0.084$ & 0.100 \\
\hline CFT87 & $19.787 \pm 0.008$ & $1.132 \pm 0.036$ & 0.130 \\
\hline CFT90 & $23.307 \pm 0.106$ & $1.315 \pm 0.718$ & 0.250 \\
\hline CFT96 & $22.517 \pm 0.047$ & $1.778 \pm 0.328$ & 0.110 \\
\hline CFT97 & $19.980 \pm 0.008$ & $0.870 \pm 0.023$ & 0.100 \\
\hline CFT101 & $22.627 \pm 0.132$ & $0.779 \pm 0.160$ & 0.100 \\
\hline CFT102 & $21.015 \pm 0.027$ & $1.379 \pm 0.273$ & 0.100 \\
\hline CFT103 & $21.703 \pm 0.035$ & $0.723 \pm 0.109$ & 0.130 \\
\hline CFT104 & $20.205 \pm 0.012$ & $0.865 \pm 0.034$ & 0.130 \\
\hline CFT105 & $19.867 \pm 0.011$ & $0.953 \pm 0.031$ & 0.100 \\
\hline CFT106 & $20.596 \pm 0.027$ & $0.968 \pm 0.061$ & 0.080 \\
\hline CFT108 & $20.544 \pm 0.027$ & $0.980 \pm 0.051$ & 0.120 \\
\hline CFT109 & $21.274 \pm 0.056$ & $1.178 \pm 0.097$ & 0.110 \\
\hline CFT110 & $21.328 \pm 0.075$ & $0.842 \pm 0.089$ & 0.100 \\
\hline CFT111 & $21.011 \pm 0.031$ & $0.901 \pm 0.062$ & 0.100 \\
\hline
\end{tabular}


TABLE 1-Continued

\begin{tabular}{cccc}
\hline \hline ID $^{\mathrm{a}}$ & $\mathrm{V}(\mathrm{mag})$ & $B-V(\mathrm{mag})$ & $E_{B-V}(\mathrm{mag})$ \\
\hline CFT112 & $22.236 \pm 0.111$ & $0.643 \pm 0.106$ & 0.110 \\
CFT113 & $20.192 \pm 0.014$ & $1.065 \pm 0.058$ & 0.070 \\
CFT114 & $20.262 \pm 0.020$ & $0.658 \pm 0.066$ & 0.100 \\
\hline
\end{tabular}

${ }^{a}$ SBKHP identifications are from Schroder et al. (2002); CFT identifications are from Chandar, Ford, \& Tsvetanov (2001); The others are from Perelmuter, Brodie, \& Huchra (1995). 
TABLE 2

MEtallicities FOR SOME SAMPLE GLOBULAR Clusters

\begin{tabular}{cccc}
\hline \hline ID & {$[\mathrm{Fe} / \mathrm{H}]$} & ID & {$[\mathrm{Fe} / \mathrm{H}]$} \\
\hline Id30244 & $-1.76 \pm 1.78$ & Is60045 & $-1.03 \pm 0.97$ \\
Is40083 & $-1.29 \pm 0.80$ & Id70319 & $-2.31 \pm 1.69$ \\
Is40165 & $-1.57 \pm 0.43$ & Id70349 & $-2.41 \pm 1.15$ \\
Is40181 & $0.64 \pm 1.43$ & Is80172 & $-0.77 \pm 0.68$ \\
Is50037 & $-2.34 \pm 0.83$ & Is90103 & $-2.23 \pm 0.99$ \\
Is50225 & $-0.04 \pm 0.59$ & SBKHP1 & $-1.207 \pm 0.369$ \\
Is50233 & $-1.75 \pm 1.02$ & SBKHP2 & $-0.707 \pm 0.167$ \\
Is50286 & $-0.04 \pm 1.85$ & SBKHP3 & $-0.211 \pm 0.193$ \\
Id50357 & $-3.62 \pm 2.97$ & SBKHP4 & $-0.407 \pm 0.088$ \\
Is50394 & $-1.50 \pm 1.29$ & SBKHP5 & $-1.086 \pm 0.091$ \\
Id50401 & $-0.04 \pm 1.00$ & SBKHP6 & $-1.493 \pm 0.206$ \\
Id50415 & $-1.90 \pm 0.71$ & SBKHP7 & $-0.955 \pm 0.098$ \\
Id50552 & $0.98 \pm 2.04$ & SBKHP8 & $-0.698 \pm 0.058$ \\
Is50696 & $-1.86 \pm 0.50$ & SBKHP9 & $-1.212 \pm 0.133$ \\
Id50785 & $-0.72 \pm 1.17$ & SBKHP10 & $-1.322 \pm 0.356$ \\
Id50826 & $-1.46 \pm 1.11$ & SBKHP11 & $-1.114 \pm 0.409$ \\
Is50861 & $-1.71 \pm 1.00$ & SBKHP13 & $-1.055 \pm 0.062$ \\
Is50886 & $-1.79 \pm 0.87$ & SBKHP14 & $-1.107 \pm 0.074$ \\
Id50960 & $-1.79 \pm 0.64$ & SBKHP15 & $-1.014 \pm 0.713$ \\
Is51027 & $-2.47 \pm 1.01$ & SBKHP16 & $-0.674 \pm 0.044$ \\
\hline
\end{tabular}

